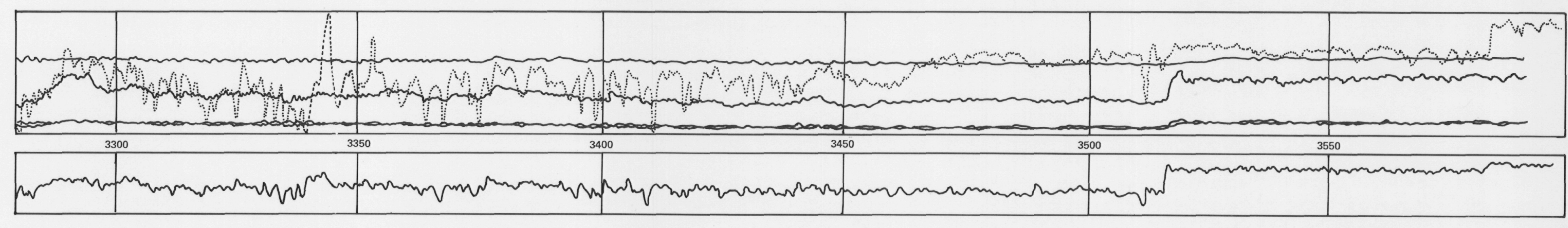

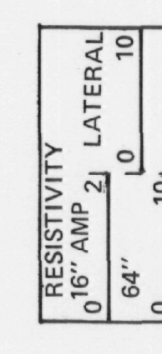

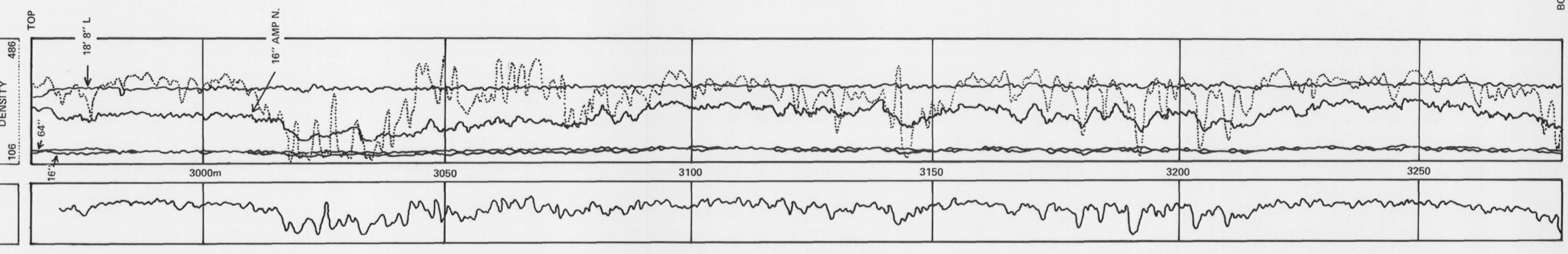

Figure 1. Composite electric log, gamma gamma density log and natural gamma radiation log for Hole 1.

$323-324$ 


\title{
9. GRAIN SIZE ANALYSIS
}

\author{
Deep Sea Drilling Project East Coast Repository \\ Lamont-Doherty Geological Observatory, Palisades, N. Y.
}

\begin{abstract}
METHOD
The methods used for grain size analysis were those laid down in the project manual and are based on standard procedures. They consist essentially of dispersing the sediment in Calgon solution (or other chemicals if necessary) using an ultrasonic probe, sieving the sand fraction, and pipetting the silt and clay fractions.
\end{abstract}

\section{SAMPLING}

Approximately 10 cubic centimeter samples were taken from the cores at about 150 centimeter intervals; that was one sample every section. Sampling at such wide intervals failed to reveal many of the finer textural variations and, in addition, the sample for analysis was usually taken from the finer portion of each bed. In several sediment types this bias in sampling did not matter. In turbidites it made a considerable difference since, as at some sites on Leg 1, turbidites formed an important part of the succession. To overcome this inadequate sampling of the turbidites, detailed sampling was made on two turbidite beds from two sites with the textural analysis being carried out by Conoco. It must be borne in mind, therefore, when examining these results that there is a bias to the fine fraction.

\section{RESULTS}

The complete list of results is given as Table 1 and the breakdown of the coarse fraction as Table 2 . Results from each sample are also plotted on the detailed core logs. Triangle diagrams based on the results for each site are given as Figure 1.

\section{Site 1}

The piston core from Site 1 consists predominantly of clay with 15 to 20 per cent silt and about 1 per cent sand. A few silt or silty clay bands occur from 450 centimeters down (Sections 4 and 5). These sediments are classified by Beall as "pelagites". Drill Cores 1 and 2 consist almost equally of silty clay and clayey silt with some silt bands. These sediments are classified as "laminites" and the grain size analyses illustrate these alternations of sediment type. Cores 3 through 9 are all silty clays on the basis of grain size analyses, though according to Beall's classification, Cores 3 through 5 are laminites and 6 through 9 are "hemi-pelagic" sediments. The sparcity of samples used in the analyses is probably the reason why this distinction is not brought out. It is noted that on grain size analysis Core 9, Section 7 shows a higher silt content ( 41 per cent) and some sand coming in. It is possible that this may mark a return to the laminite facies seen higher up, as this sediment type is not typical of the hemi-pelagics.

\section{Site 2}

At Site 2 the three samples from Core 1 are all clay with 10 to 14 per cent silt. They also show a decrease in sand content downwards from 9.5 to 2.6 per cent; this coarse fraction consists almost entirely of pelagic foraminifera and Radiolaria. In Core 2, there is a great increase in the silt fraction and the sample becomes a clayey silt. This supports the shipboard observation that there is a lower rate of clay influx from this core down. Core 3 shows an increase in silt content from 13 to 29 per cent, the sediment changes from a clay to a silty clay and the sand also increases from 4 to 9 per cent. Core 4 is comprised of clay only, with silt varying from 12 to 20 per cent.

All of the sand from Site 2 is comprised of 95 to 99 per cent fine sand with the balance falling in the medium sand grade.

\section{Site 3}

Sediments from Site 3 consist predominantly of silty clay with common clay and less commonly clayey silt. Cores 1 and 2 consist of clay or silty clay with 20 to 30 per cent silt; these correspond to the laminite facies interpretation suggested by Beall. Cores 3 through 8 are also mainly silty clays. According to the facies interpretation these are of turbidite origin, but since only the upper parts of the turbidites were sampled, the samples only contain 26 to 39 per cent silt. The clays in Cores 3 through 8 contain 10 to 20 per cent silt, and these are coccolith oozes of pelagic origin. The clayey silts in Cores 3 through 8 contain 31 to 55 per cent silt and are of turbidite origin. Core 9 contains clays with 12 to 18 per cent silt. Cores 10 to 11 are both silty clays with 32 to 26 per cent silt and indicate a return to the laminite facies.

\section{Site 4}

A variety of sediment types were found at Hole 4. These sediments range from sand to clay and represent both turbidite and pelagic sedimentation. Core 1 shows clay of pelagic origin and silty clay, and sand of 

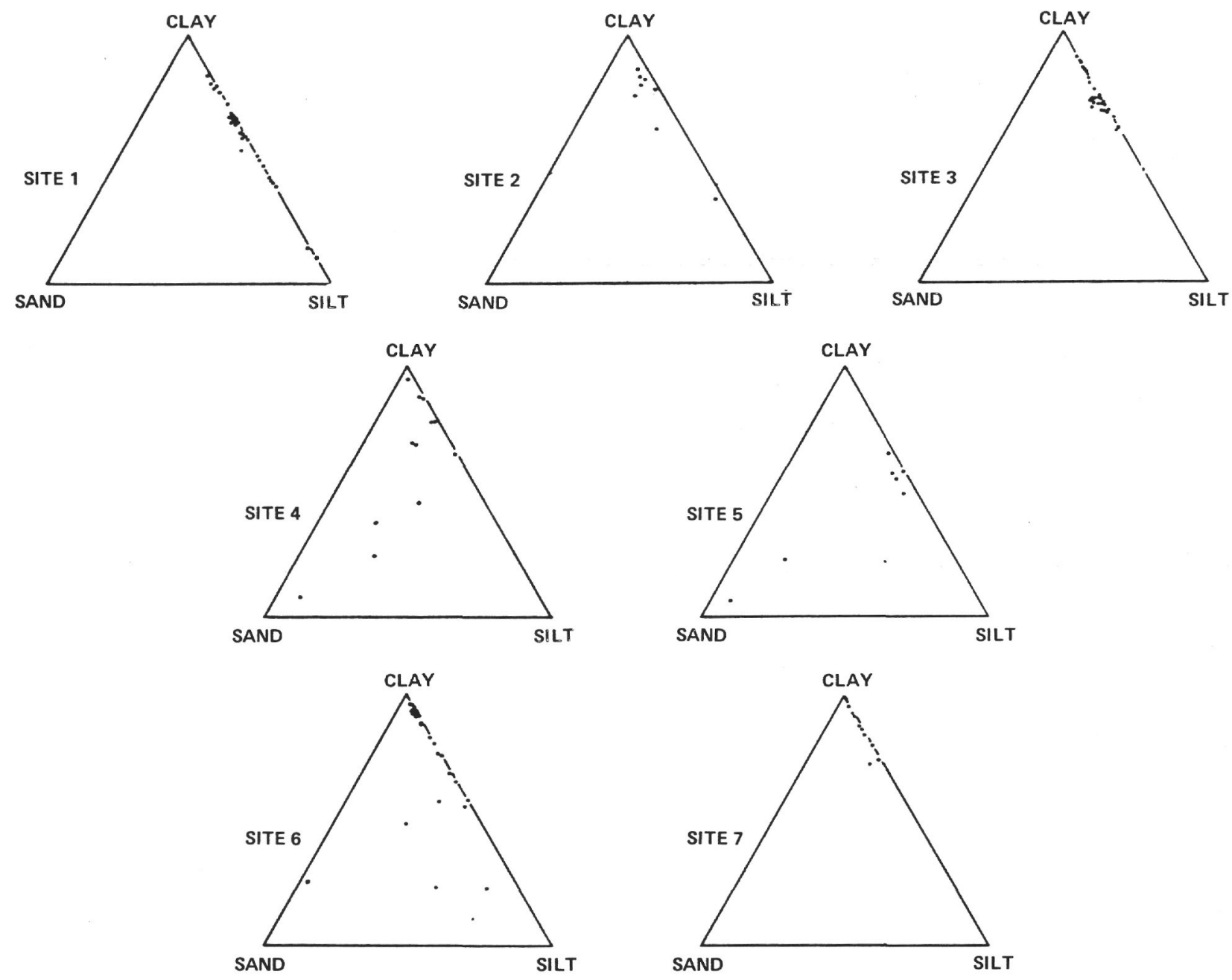

Figure 1. Triangle Diagrams of Grain Size from Leg 1.

turbidite origin. Cores 2, 3, and 4 consist of silty clay passing down into clay representing coccolith ooze. According to the core descriptions, parts of Cores 2 through 4 also contain pebbly mudstones and limestone clasts of turbidite origin. The grain size analyses do not show this unless the finer portion of the turbidite is represented by the silty clay in Core 2 . Hole 4A contains sand-silt-clay, silty clay, and clayey sand; and, this variety of coarser sediments represents turbidite deposits with only 24 to 55 per cent clay.

Three quarters of the sand fraction in Hole 4 falls into the medium size grade, and the remainder in the fine sand range. At Hole 4A, the sand is almost entirely in the fine sand grade.

\section{Site 5}

Site 5 contains sediments ranging from sand through clayey sand to silty clay which are of turbidite origin.
Some coccolith oozes are recorded in the core descriptions, but these are not in evidence from the samples taken for grain size. Two samples in Core 1 contain 87 and 60 per cent sand. Of this sand, the former is predominantly fine sand with some medium sand and in the latter about half is coarse sand with equal amounts of medium and fine sand.

\section{Site 6}

This site contains sediments ranging from clayey sand through sand-silt-clay to silty clay to clay, and these represent both turbidite and pelagic influences. Cores 1, 2 , and 3 consist almost entirely of brown clay of pelagic origin, but there is a progressive increase in the silt content with depth from 8 to 20 per cent, and at the bottom of Core 3 there is a more rapid increase to 43 per cent silt in a silty clay sample. According to the core descriptions, Cores 2 and 3 also contain mud pebble conglomerates; these do not show up in the grain size analysis. Furthermore, in Core 3, at least, 
TABLE 1

Leg 1 Grain Size Results

\begin{tabular}{|c|c|c|c|c|c|c|c|}
\hline Hole & Core & Section & $\begin{array}{l}\text { Sampled } \\
\text { at }(\mathrm{cm})\end{array}$ & $\%$ Sand & $\%$ Silt & $\%$ Clay & Classification \\
\hline 1P & 1 & 1 & 16 & 0.2 & 20.3 & 79.5 & Clay \\
\hline $1 \mathrm{P}$ & 1 & 1 & $135-137$ & 1.2 & 15.3 & 83.5 & Clay \\
\hline $1 \mathbf{P}$ & 1 & 2 & $131-133$ & 1.1 & 20.2 & 78.7 & Clay \\
\hline 1P & 1 & 3 & $114-116$ & 1.3 & 18.2 & 80.4 & Clay \\
\hline $1 P$ & 1 & 4 & 7 & 0.0 & 57.8 & 42.2 & Silty Clay \\
\hline $1 P$ & 1 & 5 & $135-137$ & 0.1 & 89.6 & 10.3 & Silt \\
\hline $1 P$ & 1 & 6 & $132-134$ & 0.2 & 22.5 & 77.3 & Clay \\
\hline 1 & 1 & 1 & $6-8$ & 0.0 & 39.5 & 60.5 & Silty Clay \\
\hline 1 & 1 & 2 & $7-9$ & 0.1 & 41.0 & 58.9 & Silty Clay \\
\hline 1 & 1 & 3 & $5.5-7.5$ & 0.0 & 33.6 & 66.4 & Silty Clay \\
\hline 1 & 1 & 4 & $7-9$ & 0.1 & 84.8 & 15.1 & Silt \\
\hline 1 & 1 & 5 & $8-10$ & 0.0 & 40.5 & 59.5 & Silty Clay \\
\hline 1 & 1 & 6 & $5-7$ & 0.3 & 58.2 & 41.5 & Clayey Silt \\
\hline 1 & 1 & 7 & $6-8$ & 0.1 & 34.6 & 65.3 & Silty Clay \\
\hline 1 & 2 & 1 & $52-54$ & 0.1 & 59.1 & 40.8 & Clayey Silt \\
\hline 1 & 2 & 2 & $3-5$ & 0.1 & 61.9 & 38.0 & Clayey Silt \\
\hline 1 & 2 & 3 & $9-11$ & 0.1 & 52.7 & 47.2 & Clayey Silt \\
\hline 1 & 3 & 1 & $7.5-9.5$ & 0.0 & 48.9 & 51.1 & Silty Clay \\
\hline 1 & 5 & 1 & $41.5-43.5$ & 0.2 & 42.0 & 57.8 & Silty Clay \\
\hline 1 & 5 & 2 & $22-24$ & 0.0 & 39.8 & 60.2 & Silty Clay \\
\hline 1 & 6 & 1 & $11-13$ & 0.2 & 34.6 & 65.2 & Silty Clay \\
\hline 1 & 6 & 2 & $9.5-11.5$ & 1.1 & 35.1 & 63.8 & Silty Clay \\
\hline 1 & 7 & 2 & $10-12$ & 0.1 & 34.7 & 65.2 & Silty Clay \\
\hline 1 & 7 & 3 & $5-7$ & 0.8 & 33.1 & 66.1 & Silty Clay \\
\hline 1 & 7 & 4 & $3-5$ & 0.6 & 38.6 & 60.8 & Silty Clay \\
\hline 1 & 7 & 5 & $8-10$ & 0.1 & 30.8 & 69.1 & Silty Clay \\
\hline 1 & 7 & 6 & 8-10 & 0.0 & 32.5 & 67.5 & Silty Clay \\
\hline
\end{tabular}


TABLE 1 - Continued

\begin{tabular}{|c|c|c|c|c|c|c|c|}
\hline Hole & Core & Section & $\begin{array}{c}\text { Sampled } \\
\text { at }(\mathrm{cm})\end{array}$ & $\%$ Sand & $\%$ Silt & $\%$ Clay & Classification \\
\hline 1 & 7 & 7 & $8-10$ & 0.1 & 33.1 & 66.8 & Silty Clay \\
\hline 1 & 8 & 2 & $13-15$ & 0.5 & 31.3 & 68.2 & Silty Clay \\
\hline 1 & 8 & 3 & $10-12$ & 2.0 & 30.6 & 67.4 & Silty Clay \\
\hline 1 & 8 & 4 & $12-14$ & 1.1 & 31.8 & 67.1 & Silty Clay \\
\hline 1 & 8 & 5 & $5-7$ & 0.3 & 35.1 & 64.6 & Silty Clay \\
\hline 1 & 8 & 6 & $10-12$ & 0.2 & 32.7 & 67.0 & Silty Clay \\
\hline 1 & 8 & 7 & $14-16$ & 0.1 & 33.4 & 66.5 & Silty Clay \\
\hline 1 & 9 & 2 & 24 & 0.2 & 31.8 & 68.0 & Silty Clay \\
\hline 1 & 9 & 3 & $11-13$ & 0.3 & 30.7 & 69.0 & Silty Clay \\
\hline 1 & 9 & 4 & $11-13$ & 0.2 & 30.6 & 69.2 & Silty Clay \\
\hline 1 & 9 & 5 & $10-12$ & 0.4 & 32.5 & 67.1 & Silty Clay \\
\hline 1 & 9 & 6 & $7-9$ & 0.1 & 27.5 & 72.4 & Silty Clay \\
\hline 1 & 9 & 7 & $7-9$ & 4.3 & 41.8 & 53.9 & Silty Clay \\
\hline 2 & 1 & 2 & $13-15$ & 9.5 & 12.0 & 76.5 & Clay \\
\hline 2 & 1 & 3 & $9-11$ & 6.1 & 14.3 & 79.6 & Clay \\
\hline 2 & 2 & 1 & 41.43 & 2.4 & 63.6 & 34.0 & Clayey Silt \\
\hline 2 & 3 & 1 & $104-106$ & 4.2 & 12.8 & 83.0 & Clay \\
\hline 2 & 3 & 2 & $5-7$ & 2.1 & 15.9 & 82.0 & Clay \\
\hline 2 & 3 & 3 & $11-13$ & 9.0 & 28.9 & 62.1 & Silty Clay \\
\hline 2 & 4 & 1 & $47-49$ & 1.4 & 20.7 & 77.9 & Clay \\
\hline 2 & 4 & 2 & $14-16$ & 2.5 & 11.7 & 85.8 & Clay \\
\hline 3 & 1 & 1 & $28-30$ & 0.0 & 28.9 & 71.1 & Silty Clay \\
\hline 3 & 1 & 2 & $23-25$ & 0.0 & 21.0 & 79.0 & Clay \\
\hline 3 & 2 & 1 & $57-59$ & 0.0 & 28.2 & 71.2 & Silty Clay \\
\hline 3 & 2 & 2 & $40-42$ & 4.7 & 25.6 & 69.7 & Silty Clay \\
\hline 3 & 2 & 3 & $14-16$ & 0.0 & 29.7 & 70.3 & Silty Clay \\
\hline 3 & 3 & 2 & $14-16$ & 0.5 & -38.7 & 60.8 & Silty Clay \\
\hline
\end{tabular}


TABLE 1 - Continued

\begin{tabular}{|c|c|c|c|c|c|c|c|}
\hline Hole & Core & Section & $\begin{array}{l}\text { Sampled } \\
\text { at }(\mathrm{cm})\end{array}$ & $\%$ Sand & $\%$ Silt & \% Clay & Classification \\
\hline 3 & 4 & 1 & $31-33$ & 0.8 & 31.9 & 67.4 & Silty Clay \\
\hline 3 & 5 & 1 & $37-39$ & 2.8 & 23.7 & 73.5 & Silty Clay \\
\hline 3 & 5 & 2 & $24-26$ & 2.7 & 29.1 & 68.2 & Silty Clay \\
\hline 3 & 5 & 3 & $15-17$ & 3.8 & -23.8 & 72.4 & Silty Clay \\
\hline 3 & 5 & 4 & $18-20$ & 1.0 & 20.0 & 79.0 & Clay \\
\hline 3 & 5 & 5 & $26-28$ & 0.1 & 38.7 & 61.2 & Silty Clay \\
\hline 3 & 6 & 1 & $61-63$ & 3.6 & 23.8 & 72.6 & Silty Clay \\
\hline 3 & 6 & 2 & $11-13$ & 2.4 & 26.6 & 71.0 & Silty Clay \\
\hline 3 & 7 & 1 & $32-34$ & 2.1 & 24.4 & 73.5 & Silty Clay \\
\hline 3 & 7 & 2 & $1-3$ & 0.2 & 18.3 & 81.5 & Clay \\
\hline 3 & 7 & 3 & $66-68$ & 0.9 & 30.8 & 68.3 & Silty Clay \\
\hline 3 & 7 & 4 & $131-133$ & 0.0 & 26.7 & 73.3 & Silty Clay \\
\hline 3 & 8 & 1 & $101-103$ & 0.1 & 15.3 & 84.6 & Clay \\
\hline 3 & 8 & 2 & $14-16$ & 0.2 & 10.5 & 89.3 & Clay \\
\hline 3 & 8 & 3 & $11-13$ & 0.3 & 55.7 & 44.0 & Clayey Silt \\
\hline 3 & 8 & 4 & $15-17$ & 0.0 & 28.5 & 71.5 & Silty Clay \\
\hline 3 & 8 & 5 & $13-15$ & 0.3 & 14.3 & 85.4 & Clay \\
\hline 3 & 8 & 6 & $44-46$ & 0.1 & 26.4 & 73.5 & Silty Clay \\
\hline 3 & 9 & 2 & $15-17$ & 0.1 & 15.4 & 84.5 & Clay \\
\hline 3 & 9 & 3 & $10-12$ & 0.0 & 12.7 & 87.3 & Clay \\
\hline 3 & 9 & 4 & $8-10$ & 0.0 & 15.7 & 84.3 & Clay \\
\hline 3 & 9 & 5 & $20-22$ & 0.0 & 18.5 & 81.5 & Clay \\
\hline 3 & 9 & 6 & $18-20$ & 0.1 & 12.0 & 87.9 & Clay \\
\hline 3 & 9 & 7 & $14-16$ & 0.1 & 17.3 & 82.6 & Clay \\
\hline 3 & 10 & 2 & $9-11$ & 0.0 & 35.9 & 64.1 & Silty Clay \\
\hline 3 & 11 & 1 & 75 & 0.5 & 31.8 & 67.7 & Silty Clay \\
\hline 4 & 1 & 1 & $41-43$ & 84.0 & 8.6 & 7.4 & Sand \\
\hline
\end{tabular}


TABLE 1 - Continued

\begin{tabular}{|c|c|c|c|c|c|c|c|}
\hline Hole & Core & Section & $\begin{array}{l}\text { Sampled } \\
\text { at }(\mathrm{cm})\end{array}$ & $\%$ Sand & $\%$ Silt & $\%$ Clay & Classification \\
\hline 4 & 1 & 2 & $100-102$ & 2.0 & 20.0 & 78.0 & Clay \\
\hline 4 & 1 & 3 & $12-14$ & 12.8 & 17.1 & 70.1 & Silty Clay \\
\hline 4 & 1 & 4 & $13-15$ & 13.6 & 16.3 & 70.1 & Silty Clay \\
\hline 4 & 1 & 5 & $50-51$ & 0.8 & 10.8 & 88.4 & Clay \\
\hline 4 & 1 & 6 & $10-12$ & 2.2 & 19.4 & 78.4 & Clay \\
\hline 4 & 2 & 1 & $107-109$ & 0.0 & 35.2 & 64.8 & Silty Clay \\
\hline 4 & 3 & 1 & $6-8$ & 0.0 & 5.7 & 94.3 & Clay \\
\hline 4 & 4 & 1 & $74-76$ & 0.2 & 11.2 & 88.6 & Clay \\
\hline $4 \mathrm{~A}$ & 1 & 1 & $11-13$ & 51.9 & 24.4 & 23.7 & Sand-Silt-Clay \\
\hline $4 \mathrm{~A}$ & 1 & 3 & $2-4$ & 12.9 & 31.2 & 55.9 & Silty Clay \\
\hline $4 \mathrm{~A}$ & 2 & 1 & $34-36$ & 43.5 & 19.0 & 37.5 & Clayey Sand \\
\hline 5 & 1 & 1 & $9-11$ & 4.8 & 46.5 & 48.7 & Silty Clay \\
\hline 5 & 1 & 2 & $12-14$ & 86.9 & 6.9 & 6.2 & Sand \\
\hline 5 & 1 & 2 & $60-62$ & 2.0 & 33.2 & 64.8 & Silty Clay \\
\hline 5 & 1 & 3 & $16-18$ & 4.1 & 41.1 & 54.8 & Silty Clay \\
\hline 5 & 1 & 3 & $75-77$ & 59.5 & 18.1 & 22.4 & Clayey Sand \\
\hline 5 & 2 & 1 & $85-87$ & 0.1 & 41.5 & 58.4 & Silty Clay \\
\hline 5 & 3 & 1 & $60-62$ & 4.4 & 38.4 & 57.2 & Silty Clay \\
\hline 6 & 1 & 1 & $24-26$ & 0.0 & 6.7 & 93.3 & Clay \\
\hline 6 & 1 & 2 & $24-26$ & 0.0 & 7.0 & 93.0 & Clay \\
\hline 6 & 1 & 3 & $9-11$ & 1.1 & 6.1 & 92.8 & Clay \\
\hline 6 & 1 & 4 & $7-9$ & 0.2 & 17.3 & 82.5 & Clay \\
\hline 6 & 2 & 1 & $44-46$ & 0.5 & 8.9 & 90.6 & Clay \\
\hline 6 & 2 & 2 & $20-22$ & 0.4 & 11.8 & 87.8 & Clay \\
\hline 6 & 2 & 3 & $11-13$ & 0.4 & 9.4 & 90.2 & Clay \\
\hline 6 & 2 & 4 & $16-18$ & 1.7 & 7.0 & 91.3 & Clay \\
\hline 6 & 2 & 5 & $25-27$ & 0.5 & 8.5 & 91.0 & Clay \\
\hline
\end{tabular}


TABLE 1 - Continued

\begin{tabular}{|c|c|c|c|c|c|c|c|}
\hline Hole & Core & Section & $\begin{array}{c}\text { Sampled } \\
\text { at }(\mathrm{cm})\end{array}$ & $\%$ Sand & $\%$ Silt & \% Clay & Classification \\
\hline 6 & 2 & 6 & $14-16$ & 1.3 & 7.4 & 91.3 & Clay \\
\hline 6 & 3 & 2 & $24-26$ & 0.1 & 24.7 & 75.2 & Clay \\
\hline 6 & 3 & 3 & $51-53$ & 0.0 & 20.4 & 79.6 & Clay \\
\hline 6 & 3 & 4 & $8-10$ & 2.0 & 43.1 & 54.9 & Silty Clay \\
\hline 6 & 4 & 1 & $29-31$ & 26.0 & 26.0 & 48.0 & Sand-Silt-Clay \\
\hline 6 & 4 & 2 & $10-12$ & 9.9 & 33.0 & 57.1 & Silty Clay \\
\hline 6 & 4 & 3 & $94-96$ & 0.2 & 34.7 & 65.1 & Silty Clay \\
\hline 6 & 5 & 1 & $13-15$ & 0.3 & 24.0 & 75.7 & Clay \\
\hline 6 & 5 & 2 & $20-22$ & 0.9 & 3.8 & 95.3 & Clay \\
\hline 6 & 6 & 1 & $11-15$ & 29.7 & 47.8 & 22.5 & Sand-Silt-Clay \\
\hline 6 & 6 & 1 & $81-83$ & 72.0 & 2.4 & 25.6 & Clayey Sand \\
\hline 6 & 6 & 2 & $3-5$ & 0.0 & 42.3 & 57.7 & Silty Clay \\
\hline $6 \mathrm{~A}$ & 1 & 1 & $33-35$ & 0.1 & 7.2 & 92.7 & Clay \\
\hline $6 \mathrm{~A}$ & 1 & 2 & $20-22$ & 0.1 & 5.1 & 94.8 & Clay \\
\hline $6 \mathrm{~A}$ & 1 & 4 & $29-31$ & 0.0 & 7.6 & 92.4 & Clay \\
\hline $6 \mathrm{~A}$ & 1 & 6 & $18-20$ & 0.0 & 6.2 & 93.8 & Clay \\
\hline 7 & 1 & 1 & $29-30$ & 0.6 & 25.5 & 73.9 & Silty Clay \\
\hline 7 & 1 & 2 & $29-30$ & 0.1 & 15.9 & 84.0 & Clay \\
\hline 7 & 1 & 3 & $12-14$ & 0.0 & 7.5 & 92.5 & Clay \\
\hline 7 & 1 & 4 & $14-16$ & 0.1 & 8.7 & 91.2 & Clay \\
\hline 7 & 1 & 6 & 19-21 & 0.1 & 4.8 & 95.1 & Clay \\
\hline $7 \mathrm{~A}$ & 2 & 1 & $26-28$ & 0.0 & 20.3 & 79.7 & Clay \\
\hline $7 \mathrm{~A}$ & 2 & 2 & $21-23$ & 0.4 & 12.2 & 87.4 & Clay \\
\hline $7 \mathrm{~A}$ & 3 & 1 & $30-32$ & 0.3 & 12.8 & 86.9 & Clay \\
\hline $7 \mathrm{~A}$ & 3 & 2 & $12-14$ & 4.9 & 23.2 & 72.9 & Clay \\
\hline
\end{tabular}


TABLE 2

Leg 1 Coarse Fraction Results

\begin{tabular}{ccccc|ccc}
\hline & & & & & \multicolumn{3}{c}{ Size distribution of sand } \\
\cline { 5 - 7 } Hole & Core & Section & $\begin{array}{c}\text { Sampled } \\
\text { at }(\mathrm{cm})\end{array}$ & $\begin{array}{c}\text { \% Sand in } \\
\text { Sediment }\end{array}$ & $\begin{array}{c}\text { Coarse } \\
(>2.0 \mathrm{~mm})\end{array}$ & $\begin{array}{c}\text { Medium } \\
(2.0-0.5 \mathrm{~mm})\end{array}$ & $\begin{array}{c}\text { Fine } \\
(0.5-0.062 \mathrm{~mm})\end{array}$ \\
\hline 2 & 1 & 2 & $13-15$ & 9.5 & 0.0 & 4.5 & 95.5 \\
2 & 1 & 3 & $9-11$ & 6.1 & 0.0 & 0.5 & 99.5 \\
2 & 3 & 3 & $11-13$ & 9.0 & 0.0 & 4.4 & 95.6 \\
4 & 1 & 1 & $41-43$ & 84.0 & 0.6 & 85.5 & 13.9 \\
4 & 1 & 3 & $12-14$ & 12.8 & 2.0 & 76.0 & 22.0 \\
4 & 1 & 4 & $13-15$ & 13.6 & 0.0 & 79.9 & 21.1 \\
$4 \mathrm{~A}$ & 1 & 1 & $11-13$ & 51.9 & 0.0 & 0.8 & 99.2 \\
$4 \mathrm{~A}$ & 1 & 3 & $2-4$ & 12.9 & 0.0 & 0.0 & 100.0 \\
$4 \mathrm{~A}$ & 2 & 1 & $34-36$ & 43.5 & 0.0 & 6.1 & 93.9 \\
5 & 1 & 2 & $12-14$ & 86.9 & 1.9 & 21.4 & 76.7 \\
5 & 1 & 3 & $75-77$ & 59.5 & 58.6 & 24.3 & 17.1 \\
6 & 4 & 1 & $29-31$ & 26.0 & 18.1 & 65.5 & 16.4 \\
6 & 4 & 2 & $10-12$ & 9.9 & 0.0 & 0.0 & 100.0 \\
6 & 6 & 1 & $11-15$ & 99.7 & 0.0 & 0.3 & 99.7 \\
\hline
\end{tabular}


these are probably artifacts of drilling. Cores 4,5 , and 6 represent an admixture of turbidite constituents with diatom-coccolith laminites and clay-rich diatom ooze. Core 5 samples are only clays.

From Section 1, Core 6, two samples were taken for analysis; one was taken from the upper part of a turbidite deposit, and the other from the upper lower part, and these were respectively, sand-silt-clay and clayey sand. Details of the analysis can be seen in Table 1. It will be noted that both contain about 25 per cent clay, but the remainder of the sediment in the lower part of the turbidite bed consists almost entirely of sand, while the upper part contains more silt than sand. Detailed textural analyses of 12 samples from this turbidite were made by Conoco, and the results are given in Chapter 24. The sand fraction in this turbidite falls entirely in the fine sand grade. The samples from $6 \mathrm{~A}$ are all clay with a maximum of 7 per cent silt.

The sand in Core 4, Section 1 is predominantly medium-grained, with almost equal amounts of coarse and fine sand forming the remaining third.

\section{Site 7}

With the exception of one sample, all the analyses of samples from Site 7 are clays with approximately 5 to 20 per cent silt; these are pelagic brown clays. One sample is a silty clay, but it still contained 73.9 per cent clay. The 25.5 per cent silt and minute part of sand are of organic origin, as the sample was collected near the top of the hole where foraminiferal-coccolith oozes occur. Some turbidites were recorded also, but it appears that none of these were sampled for grain size analysis. 